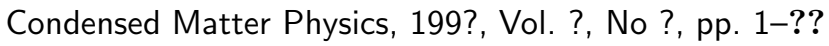

\title{
On the theory of phase transitions in binary fluid mixtures
}

\author{
O.V.Patsahan \\ Institute for Condensed Matter Physics of the National Academy of Sciences \\ of Ukraine, 1 Svientsitskii Str., UA-290011, Lviv, Ukraine
}

November 4, 2018

\begin{abstract}
The microscopic approach to the description of the phase behaviour and critical phenomena in binary fluid mixtures is proposed. It is based on the method of collective variables with a reference system. The physical nature of the order parameter in a binary mixture is discussed. The basic density measure (GinsburgLandau-Wilson Hamiltonian) is obtained in the collective variable phase space which contains the variable connected with the order parameter of the system. It is shown that the problem can be reduced to the 3D Ising model in an external field.
\end{abstract}

Key words: phase transition, binary mixture, order parameter

PACS: 05.70.Fh, 05.70.Jk, 64.10. $+h$

\section{Introduction}

The study of phase transitions and critical phenomena in multicomponent fluid systems is very interesting from the theoretical as well as practical point of view. Whereas in one-component fluid systems only gas-liquid equilibria exist,three different types of two-phase equilibria have to be considered in fluid mixtures: gas-liquid, liquid-liquid and gas-gas equilibria. Despite the numerous experimental results now available [ 1]- [ 5], theoretical achievements in understanding a microscopic mechanism of phase behaviour and nonuniversal critical properties of multicomponent fluids are limited. Most of the theoretical studies devoted to this problem may be divided into three main groups: phenomenological theories, mean field approaches and integral equation methods. The phenomenological approaches [ 6]- [ 11] give predictions about critical exponents and scaling functions but no quantitative estimates of nonuniversal critical amplitudes are possible within this framework. The problem of the phase diagram sensitivity to the microscopic model also remains unsolved. The mean field theories [12] and integral equation methods [ 13]- reproduce different phase diagram types by varying the microscopic parameters but give qualitative estimates. 
Of special interest are Refs.[22]- [27] devoted to the study of both the universal and nonuniversal properties. They are based on the previously proposed approach to the study of the gas-liquid critical point in a one-component fluid., namely, the hierarchical reference theory (HRT) [28]. On the microscopic Hamiltonian grounds, the HRT develops the renormalization group structure near a critical point. Recently this approach was also used for studying the 3D Ising model [29].

In spite of the doubtless success of the HRT the problem remains of constructing a theory that allows within a unified approach a complete description of the phase behaviour of multicomponent continuous systems beginning with the Hamiltonian and ending with the thermodynamic functions in the neighbourhood of the phase transition point. This program has already been accomplished in both the 3D Ising model [32] and a simple fluid near the gas-liquid critical point [33]-[35]. Within the framework of the $\phi^{4}$ model this approach has permitted to obtain the non-classical critical exponents and analytical expressions for thermodynamic functions .

This theory has its origin in the approach based on the functional representation of a partition function in the collective variables (CV) space [31]. First the method of CV was proposed for a study of the charged particles systems[ 30]-[31] and then it was applied to the second order phase transition theory [32]. The point is that the statistical description of the phase transition is to be performed in the appropriate phase space specific for a certain physical model. Among the independent variables of this space there must be those connected with order parameters. This phase space forms a set of $\mathrm{CV}$. Each of them is a mode of density fluctuations corresponding to the specific feature of the model under consideration. In particular, for a magnetic system the CV are variables connected with spin density fluctuation modes, for a one-component fluid - with particle density fluctuation modes. What is the content of the CV for a multicomponent system? We will answer this question below.

Experiments have shown that fluids and fluid mixtures near the ordinary critical points belong to the universality class of Ising-like systems [ 3]. Thus, a study of critical properties of multicomponent systems requires, on the one hand, an extension of the method worked out for one-component fluids and, on the other hand, their further development. In [ 36, 37] we developed the CV method with a reference system (RS) for the case of a grand canonical ensemble for a multicomponent system. Within the framework of this approach the phase diagram of a symmetrical mixture was examined in detail [ 38]-[41]. Our previous study has been mainly restricted to the Gaussian model. In [37] we obtained the explicit form of the Ginzburg-LandauWilson Hamiltonian ( $\phi^{4}$ model) for a binary symmetrical mixture in the vicinity of the gas-gas demixing critical point. In this paper we generalize the approach in the case of a non-symmetrical binary fluid system (the system of different size particles interacting via different potentials).

The layout of the paper is as follows. We give a functional representation of a grand partition function of a two-component system in Section 2. The physical nature of the order parameter in a binary mixture is discussed in Section 3. In Section 4 we construct the basic density measure (Ginzburg-Landau-Wilson Hamiltonian) with respect to the $\mathrm{CV}$ which include a variable corresponding to the order param- 
eter.

\section{Functional representation of the grand partition function of a binary mixture}

Let us consider a classical two-component system of interacting particles consisting of $N_{a}$ particles of species $a$ and $N_{b}$ particles of species $b$. The system is in volume $V$ at temperature $T$.

Let us assume that an interaction in the system has a pairwise additive character. The interaction potential between a $\gamma$ particle at $\vec{r}_{i}$ and a $\delta$ particle at $\vec{r}_{j}$ may be expressed as a sum of two terms:

$$
U_{\gamma \delta}\left(r_{i j}\right)=\psi_{\gamma \delta}\left(r_{i j}\right)+\phi_{\gamma \delta}\left(r_{i j}\right),
$$

where $\psi_{\gamma \delta}(r)$ is a potential of a short-range repulsion that will be chosen as an interaction between two hard spheres $\sigma_{\gamma \gamma}$ and $\sigma_{\delta \delta} . \phi_{\gamma \delta}(r)$ is an attractive part of the potential which dominates at large distances. An arbitrary positive function belonging to the $L_{2}$ class can be chosen as the potential $\phi_{\gamma \delta}(r)$.

Further consideration of the problem is done in the extended phase space: in the phase space of the Cartesian coordinates of the particles and in the CV phase space. An interaction connected with repulsion (potential $\psi_{\gamma \delta}(r)$ ) is considered in the space of the Cartesian coordinates of the particles. We call this two-component hard spheres system a reference system (RS). The interaction connected with an attraction (potential $\phi_{\gamma \delta}(r)$ ) is considered in the CV space. The phase space overflow is cancelled by introduction of the transition Jacobian. The contribution of the short-range forces to the long-range interaction screening is ensured by averaging this Jacobian over the RS.

Then a grand partition function in the $\mathrm{CV}$ representation with a $\mathrm{RS}$ can be written as (for details see Appendix A):

$$
\Xi=\Xi_{0} \Xi_{1}
$$

where $\Xi_{0}$ is the grand partition function of the RS. The thermodynamic and structural properties of the RS are assumed to be known. Although it is known that mixtures with only repulsive interactions might undergo a phase transition [43], we assume that in the region of temperatures, concentrations and densities we are interested in, thermodynamic functions of the RS remain analytic. $\Xi_{1}$ has the following form:

$$
\begin{aligned}
\Xi_{1}= & \int(d \rho)(d c) \exp \left[\beta \mu_{1}^{+} \rho_{0}+\beta \mu_{1}^{-} c_{0}-\frac{\beta}{2 V} \sum_{\vec{k}}\left[\tilde{V}(k) \rho_{\vec{k}} \rho_{-\vec{k}}+\right.\right. \\
& \left.\left.\tilde{W}(k) c_{\vec{k}} c_{-\vec{k}}+\tilde{U}(k) \rho_{\vec{k}} c_{-\vec{k}}\right]\right] J(\rho, c) .
\end{aligned}
$$

Here the following notations are introduced:

$\rho_{\vec{k}}$ and $c_{\vec{k}}$ are the CV connected with total density fluctuation modes and relative density (or concentration) fluctuation modes in the binary system. 
Functions $\mu_{1}^{+}$and $\mu_{1}^{-}$have the form:

$$
\mu_{1}^{+}=\frac{\sqrt{2}}{2}\left(\mu_{1}^{a}+\mu_{1}^{b}\right), \quad \mu_{1}^{-}=\frac{\sqrt{2}}{2}\left(\mu_{1}^{a}-\mu_{1}^{b}\right)
$$

(the expressions for $\mu_{1}^{\gamma}$ are given in Appendix A) and are determined from the equations

$$
\begin{gathered}
\frac{\partial \ln \Xi_{1}}{\partial \beta \mu_{1}^{+}}=\langle N\rangle, \\
\frac{\partial \ln \Xi_{1}}{\partial \beta \mu_{1}^{-}}=\left\langle N_{a}\right\rangle-\left\langle N_{b}\right\rangle . \\
\tilde{V}(k)=\left(\tilde{\phi}_{a a}(k)+\tilde{\phi}_{b b}(k)+2 \tilde{\phi}_{a b}(k)\right) / 2 \\
\tilde{W}(k)=\left(\tilde{\phi}_{a a}(k)+\tilde{\phi}_{b b}(k)-2 \tilde{\phi}_{a b}(k)\right) / 2 \\
\tilde{U}(k)=\left(\tilde{\phi}_{a a}(k)-\tilde{\phi}_{b b}(k)\right) / 2, \\
J(\rho, c)=\int(d \omega)(d \gamma) \exp \left[i 2 \pi \sum_{\vec{k}}\left(\omega_{k} \rho_{k}+\gamma_{k} c_{k}\right)\right] J(\omega, \gamma), \\
J(\omega, \gamma)=\exp _{n \geq 1}\left[\sum_{i_{n} \geq 0} \frac{(-i 2 \pi)^{n}}{n !} \sum_{\vec{k}_{1} \ldots \vec{k}_{n}} M_{n}^{\left(i_{n}\right)}(0, \ldots, 0) \times\right. \\
\left.\gamma_{\vec{k}_{1}} \ldots \gamma_{\vec{k}_{i_{n}}} \omega_{\vec{k}_{i_{n+1}}} \ldots \omega_{\vec{k}_{n}}\right] .
\end{gathered}
$$

Index $i_{n}$ is used to indicate the number of variables $\gamma_{\vec{k}}$ in the cumulant expansion (2.6). Cumulants $M_{n}^{\left(i_{n}\right)}$ are expressed as linear combinations of the partial cumulants $M_{\gamma_{1} \ldots \gamma_{n}}$ (see (5.3)) and are presented for $\gamma_{1}, \ldots, \gamma_{n}=a, b$ and $n \leq 4$ in [ 37] (see Appendix B in [37]).

Formulas (2.1)-(2.7) are the initial working formulas in our study of phase transitions in binary fluids.

\section{The order parameter in a binary mixture}

A choice of the order parameter in multicomponent fluid mixtures is a serious problem because the character of the phase transition can be changing continuously from the pure gas-liquid transition to the mixing-demixing one. The question of the physical nature of the order parameter in binary fluid mixtures has been considered until recently from the point of view of both the phenomenological theory [ 10, 11] and the microscopic approach [20], [ 23], [ 37], [ 42]. Nowdays the commonly accepted idea is that both the gas-liquid and mixing-demixing phase transitions are accompanied by total density fluctuations as well as by relative density (or concentration) fluctuations. This is the only symmetrical mixture which exibits a complete distinction between these two processes [37]. However, most likely such an 
"ideal" system does not occur in reality. In real mixtures the contribution from each type of the fluctuation processes changes along the critical curve. The evaluation of such contributions at each critical curve point is essential to the definition of the order parameter and to the understanding of the phase transition character in the mixture. It seems to us that in our approach the question of the physical nature of the order parameter has a consistent and clear solution. Here we shall briefly focus on it.

Let us consider a Gaussian approximation of functional integral (2.1)-(2.7). This approximation, also known as the random-phase approximation, yields the correct qualitative picture of the phenomena under consideration. As the result of the integration over variables $\gamma_{k}$ and $\omega_{k}, \Xi_{1}$ can be rewritten as

$$
\begin{aligned}
\Xi_{1}^{G}= & \frac{1}{2 \pi} \prod_{\vec{k}}^{\prime} \frac{1}{\pi} \frac{1}{\sqrt{\Delta(k)}} \int(d \rho)(d c) \exp \left[\rho_{0}\left(\beta \mu_{1}^{+}+\aleph_{1} / \Delta\right)+\right. \\
& c_{0}\left(\beta \mu_{1}^{-}+\aleph_{2} / \Delta\right)-\left(M_{1}^{(0)} \aleph_{1}+M_{1}^{(1)} \aleph_{2}\right)-\frac{1}{2} \sum_{\vec{k}}\left[\rho_{\vec{k}} \rho_{-\vec{k}} \times\right. \\
& \left.\left.A_{11}(k)+c_{\vec{k}} c_{-\vec{k}} A_{22}(k)+2 \rho_{\vec{k}} c_{-\vec{k}} A_{12}(k)\right]\right],
\end{aligned}
$$

where

$$
\begin{aligned}
\aleph_{1}=M_{2}^{(2)} M_{1}^{(0)}-M_{2}^{(1)} & M_{1}^{(1)}, \quad \aleph_{2}=M_{2}^{(0)} M_{1}^{(0)}-M_{2}^{(1)} M_{1}^{(0)} \\
A_{11}(k) & =-\frac{1}{2}\left(\frac{\beta}{V} \tilde{V}(k)+\frac{M_{2}^{(2)}}{\Delta}\right) \\
A_{22}(k) & =-\frac{1}{2}\left(\frac{\beta}{V} \tilde{W}(k)+\frac{M_{2}^{(0)}}{\Delta}\right) \\
A_{12}(k) & =-\frac{1}{2}\left(\frac{\beta}{V} \tilde{U}(k)-\frac{M_{2}^{(1)}}{\Delta}\right) \\
\Delta & =M_{2}^{(0)} M_{2}^{(2)}-\left(M_{2}^{(1)}\right)^{2} .
\end{aligned}
$$

In order to determine the phase space of the CV connected with the order parameters we distinguish independent collective excitations by diagonalizing the square form in (3.1) by means of the orthogonal transformation:

$$
\begin{aligned}
\rho_{\vec{k}} & =A(k) \eta_{\vec{k}}+B(k) \xi_{\vec{k}} \\
c_{\vec{k}} & =C(k) \eta_{\vec{k}}+D(k) \xi_{\vec{k}} .
\end{aligned}
$$

The explicit forms for coefficients $A(k), B(k), C(k)$ and $D(k)$ are given in Appendix B.

As a result, we have

$$
\begin{aligned}
\Xi_{1}^{G}= & \frac{1}{2 \pi} \prod_{\vec{k}} \frac{1}{\pi} \frac{1}{\sqrt{\Delta(k)}} \int(d \eta)(d \xi) \exp \left[\eta_{0}\left(A M_{1}+C M_{2}\right)+\right. \\
& \xi_{0}\left(B M_{1}+D M_{2}\right)-\left(M_{1}^{(0)} \aleph_{1}+M_{1}^{(1)} \aleph_{2}\right) /(\Delta(0))- \\
& \left.\frac{1}{2} \sum_{\vec{k}}\left(\varepsilon_{11}(k) \eta_{\vec{k}} \eta_{-\vec{k}}+\varepsilon_{22}(k) \xi_{\vec{k}} \xi_{-\vec{k}}\right)\right]
\end{aligned}
$$


where

$$
\varepsilon_{i i}(k)=-\left(A_{11}(k)+A_{22}(k) \mp \sqrt{\left(A_{11}(k)-A_{22}(k)\right)^{2}+4 A_{12}^{2}(k)}\right) .
$$

One of the quantities (3.5) (or both) tends to zero as the critical temperature is approached. On the other hand, we have to find such a CV $\eta_{\vec{k}^{*}}\left(\right.$ or $\xi_{\vec{k}^{*}}$ ) which is connected with the order parameter. Index $\vec{k}^{*}$ must correspond to the point of minimum of one of the functions $\varepsilon_{11}(k)$ or $\varepsilon_{22}(k)$ ( or both). These functions depend on temperature, attractive potentials $\tilde{\phi}_{\gamma \delta}(k)$ and characteristics of the RS. The RS enters into (3.5) by cumulants $M_{\gamma \delta}(k) . M_{\gamma \delta}(k)$ can be expressed by the Fourier transforms of the direct correlation functions $C_{\gamma \delta}(k)$ by means of the OrnsteinZernike equations for a mixture. In [44] the analytic solution of the Percus-Yevick equation for a hard sphere binary mixture was obtained.

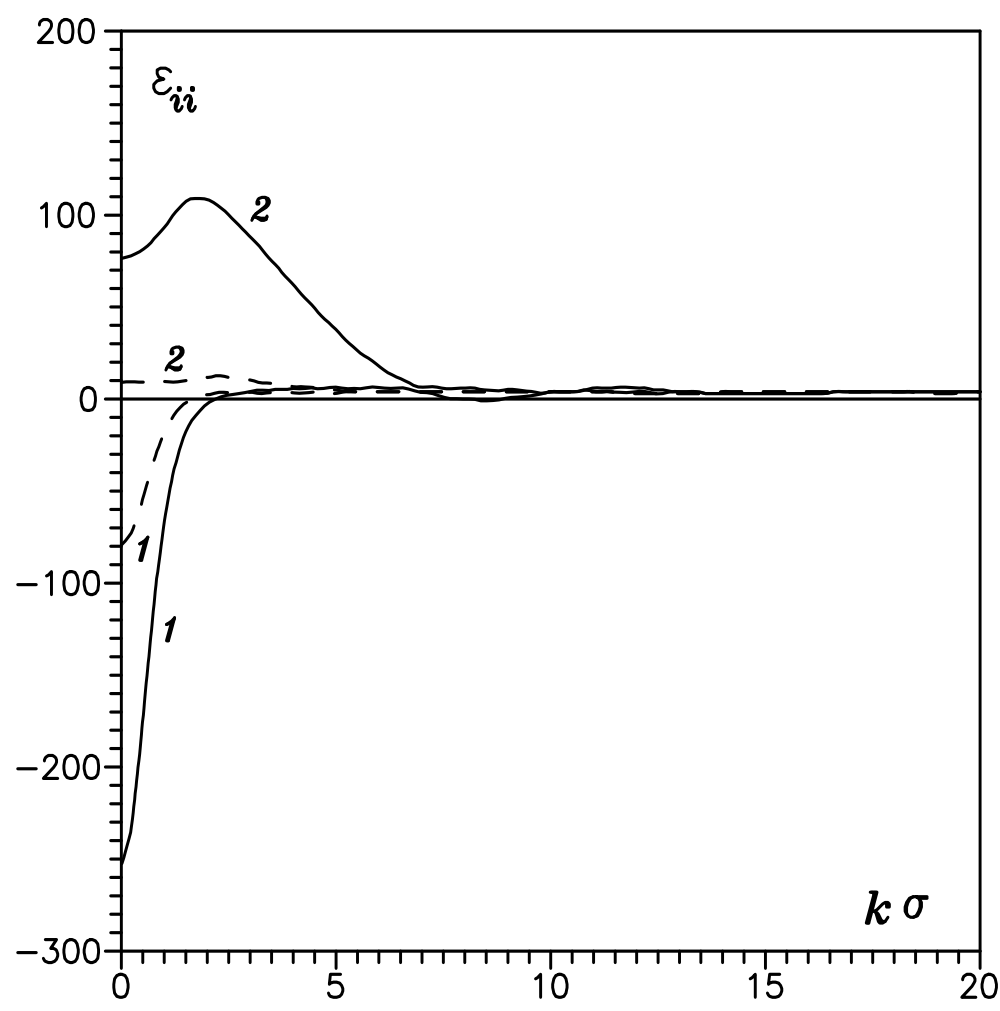

Figure 1. Coefficients $\varepsilon_{11}(k)$ and $\varepsilon_{22}(k)$ as functions of $k$ for the $\mathrm{NH}_{3}-\mathrm{N}_{2}$ mixture. The solid curves represent the gas-gas demixing critical point $\left(T=413^{\circ} \mathrm{K}, \eta=\right.$ $0.45, x=0.5)$ and the dashed curves represent the gas-liquid critical point $(T=$ $\left.373^{\circ} \mathrm{K}, \eta=0.12, x=0.5\right)$ [40]

Coefficients $\varepsilon_{11}(k)$ and $\varepsilon_{22}(k)$ are studied both as wave vector functions at different values of temperature $T$, density $\eta$ and concentration $x$ including the gas-liquid and mixing-demixing critical points (see Fig. 1) [ 40] and as temperature functions 
at $\vec{k}=0$ (see Fig. 2) [41]. The results show that branch $\varepsilon_{11}(k)$ becomes a critical one no matter whether the system approaches the gas-liquid or gas-gas demixing critical point. Moreover, $\varepsilon_{11}(k)$ and $\varepsilon_{22}(k)$ have the minima at $\vec{k}=0$ [40]. Hence we can draw the following conclusions:

1. Branch $\varepsilon_{11}(k)$ is always critical.

2. Because $\varepsilon_{11}(k)$ has the minimum at $\vec{k}=0$, the $\mathrm{CV}$ connected with the order parameter is $\eta_{0}$ in the case of the gas-liquid critical point as well as in the case of the mixing-demixing phase transition. The particular form of $\eta_{0}$ for each of these phenomena can be determined by means of the relations between the microscopic parameters, temperature, density and concentration of the system, e.g. by means of coefficients $A, B, C$ and $D$.

3. In the plane $\left(\rho_{0}, c_{0}\right)$ we have distinguished two directions: the direction of strong fluctuations $\eta_{0}$ and the direction of weak fluctuations $\xi_{0}$. As a result, we can write the conditions for the binary mixture critical point in the form:

$$
\begin{aligned}
{\left[\frac{\partial^{2} \Omega}{\partial \eta_{0}^{2}}\right]_{c} } & =0 \\
{\left[\frac{\partial^{2} \Omega}{\partial \eta_{0} \partial \xi_{0}}\right]_{c} } & =0 \\
{\left[\frac{\partial^{3} \Omega}{\partial \eta_{0}^{3}}\right]_{c} } & =0
\end{aligned}
$$

where $\Omega=-k T \ln \Xi$ is a grand canonical potential.

Now let us consider equations $(3.3)$ at $k=0$. From (3.3) it follows that

$$
\begin{aligned}
& \eta_{0}= \pm D(0) \rho_{0} \mp B(0) c_{0} \\
& \xi_{0}=\mp C(0) \rho_{0} \pm A(0) c_{0},
\end{aligned}
$$

where the upper sign corresponds to the case

$$
\left|A_{12}\right|=-A_{12} \quad(A D-B C=1)
$$

and the lower sign corresponds to

$$
\left|A_{12}\right|=A_{12} \quad(A D-B C=-1) .
$$

On the other hand, (3.9) can be rewritten as

$$
\begin{aligned}
& \eta_{0}= \pm \rho_{0} \cos \theta \pm c_{0} \sin \theta \\
& \xi_{0}=-\rho_{0} \sin \theta+c_{0} \cos \theta .
\end{aligned}
$$

Comparing (3.9) and (3.12) we can determine rotation angle $\theta$ of axes $\eta_{0}$ and $\xi_{0}$ in the $\left(\rho_{0}, c_{0}\right)$ plane from the equation

$$
\tan \theta=\frac{C}{A}
$$




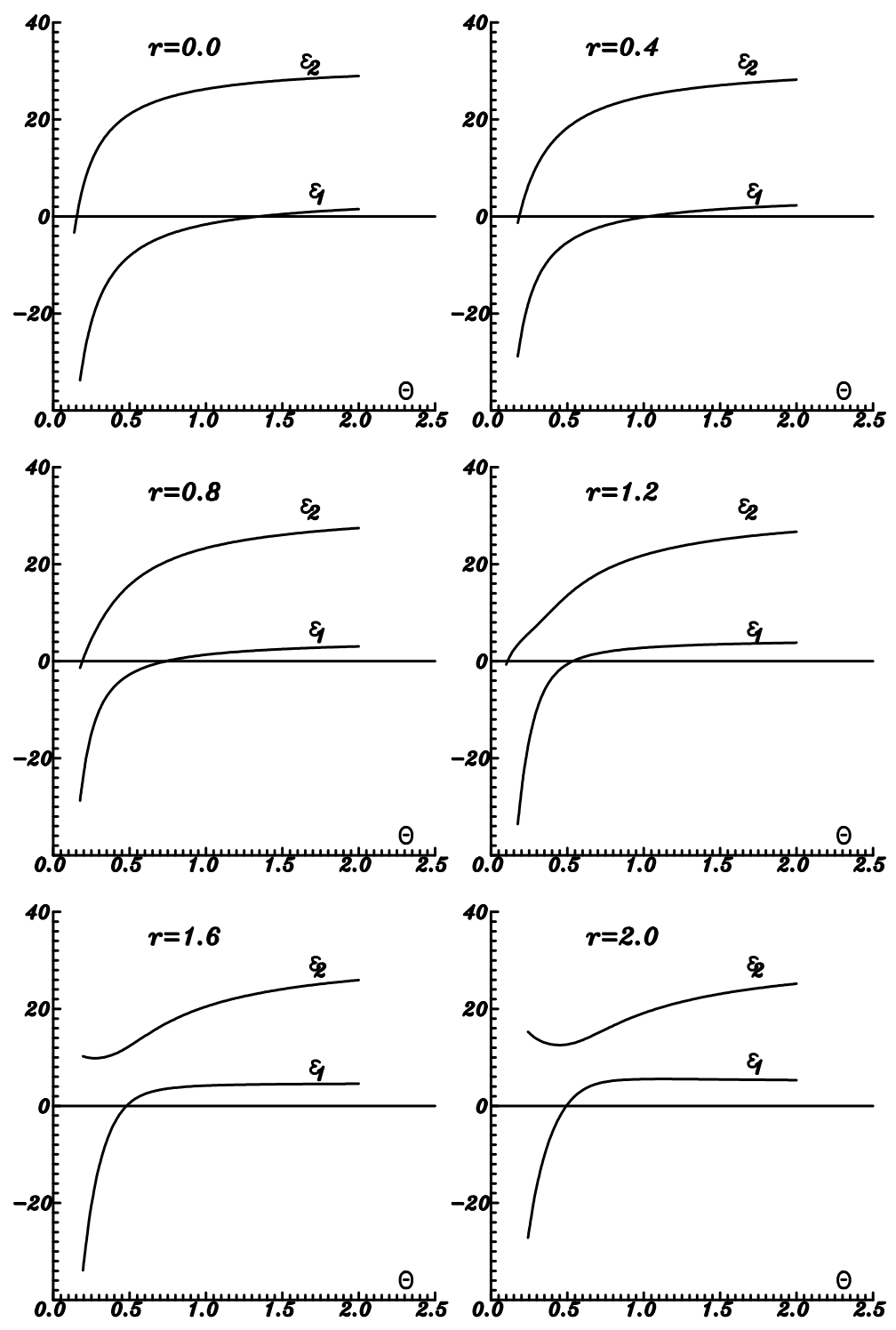

Figure 2. Coefficients $\varepsilon_{11}(k=0)$ and $\varepsilon_{22}(k=0)$ as functions of the dimensionless temperature $T$ at $q=1.0, \alpha=1.0, \eta=0.26$ and $x=0.7$ for different values of $\mathrm{r}$ $\left(\varepsilon_{i i}(k=0)\right.$ are obtained for a binary hard core Yukawa mixture [40]) 
(in both cases (3.10) and (3.11)). In the case (3.11) transformation (3.12) corresponds to both the mirror image with respect to the $c_{0}$ axis and the rotation in the $\left(\rho_{0}, c_{0}\right)$ plane. Thus, the proposed approach allows us, on microscopic grounds, to define the order parameter at each point along a critical curve and so to understand the phase transition character in the binary mixture.

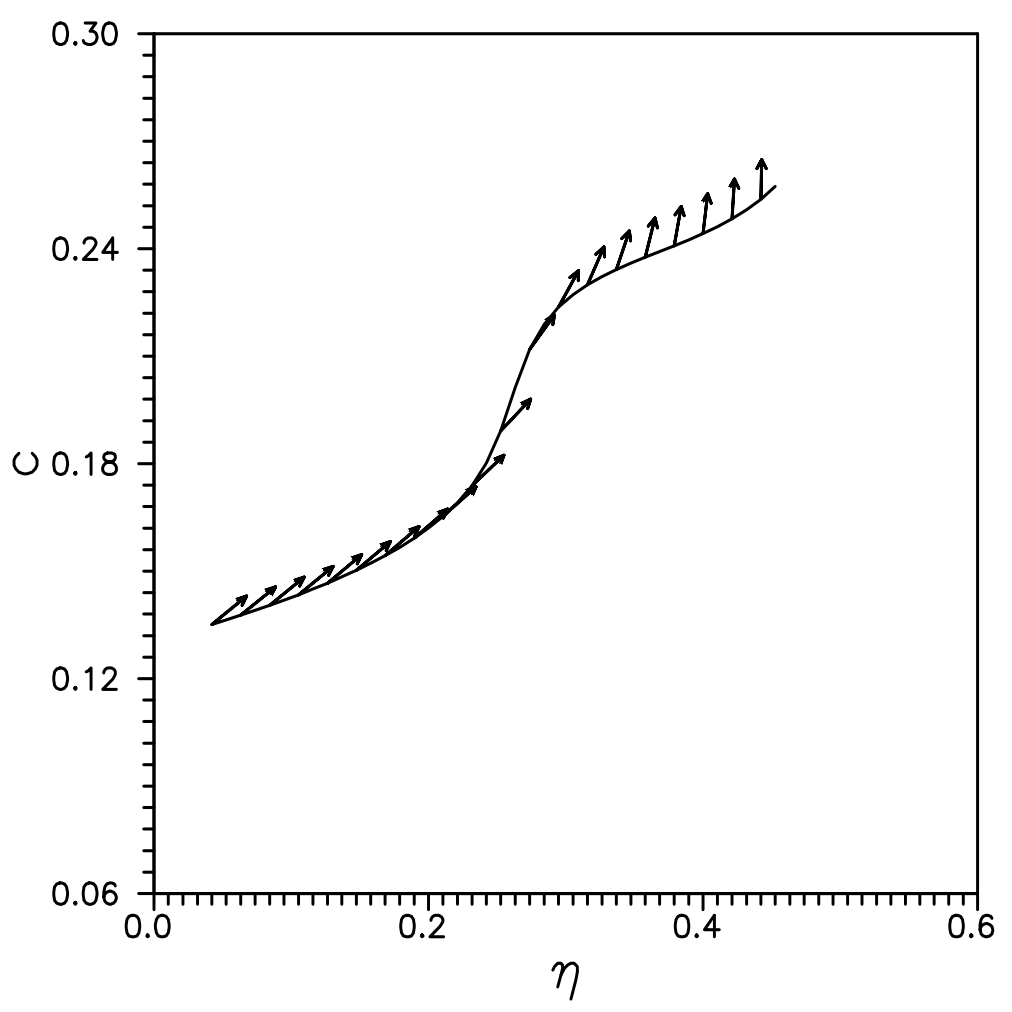

Figure 3. Density-concentration projection of the critical line of the model binary mixture at $\alpha=1.0, q=0.9$ and $r=0.6$ (mean field approximation)

Figures 3-5 show the $(\eta, x)$ projections of the $(T, \eta, x)$ critical surfaces of the model binary mixture for various values of microscopic parameters $\alpha, q$ and $r$. The arrows show the direction of the strong fluctuations (order parameter) along the critical curve in accordance with formula (3.13). Here the following notations are introduced: $\eta$ is the packing density $\left(\eta=\eta_{a}+\eta_{b}, \eta_{i}=\pi \rho_{i} \sigma_{i i}^{3} / 6, \rho_{i}=\langle N\rangle / V\right.$ is the number density of species $i), x$ is the concentration $\left(x=\left\langle N_{b}\right\rangle /\langle N\rangle\right), \alpha=\sigma_{a a} / \sigma_{b b}$ is the hard sphere ratio, $\sigma_{i i}$ is the hard sphere diameter, $q_{\sim}=-\tilde{\phi}_{b b}(\underset{\sim}{0}) /\left|\tilde{\phi}_{a a}(0)\right|$ is the dimensionless "like" interaction strength and $r=-\tilde{\phi}_{a b}(0) /\left|\tilde{\phi}_{a a}(0)\right|$ is the "unlike" interaction strength (the form of $\phi_{i j}(r)$ is not specified). The critical surface is derived from the $\left(f_{m f}, V, x\right)$ surface by the equations for a binary mixture critical point (in terms of the Helmholtz free energy [1]). $f_{m f}$ is the Helmholtz free energy of a binary mixture in the mean field approximation (see Appendix C).

The purpose of our further study is the calculation of the binary mixture be- 


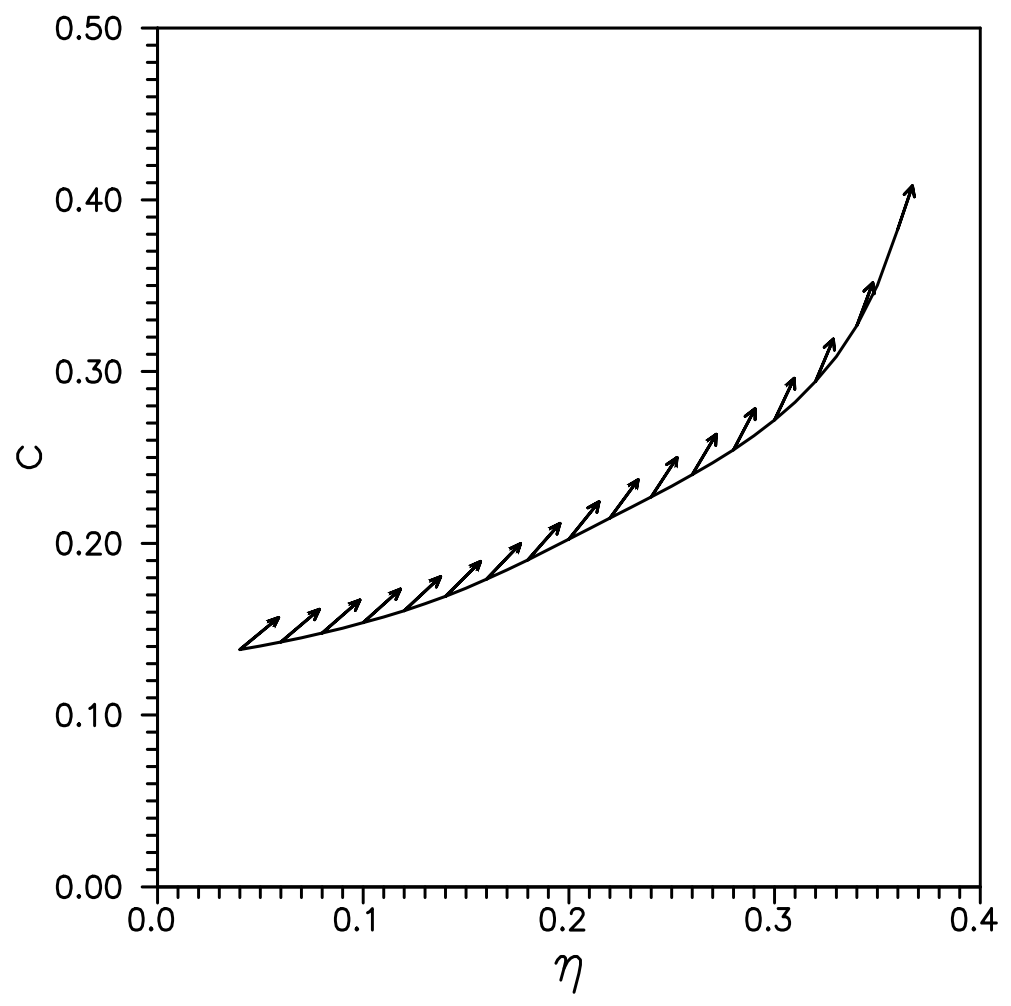

Figure 4. Same as figure 3 at $\alpha=0.9, q=0.9$ and $r=0.6$ 
haviour in the vicinity of its critical points. Based on the Gaussian distribution (3.1)-(3.2) we have determined the critical branch and, correspondingly, CV $\eta_{0}$ connected with the order parameter. Now we shall construct the basic density measure with respect to $\mathrm{CV} \eta_{\vec{k}}$. As it is shown in [32], in the vicinity of the critical point the basic density measure exists which includes higher powers of $\mathrm{CV}$ than the second power. We shall follow the program: (1) having passed from CV $\rho_{\vec{k}}$ and $c_{\vec{k}}$ to $\mathrm{CV} \eta_{\vec{k}}$ and $\xi_{\vec{k}}$ in (2.1), we shall integrate over variables $\xi_{\vec{k}}$ with the Gaussian density measure; (2) then we shall construct the basic density measure with respect to variables $\eta_{\vec{k}}$ (Ginzburg-Landau-Wilson Hamiltonian). We shall restrict our consideration to the $\eta^{4}$ model.

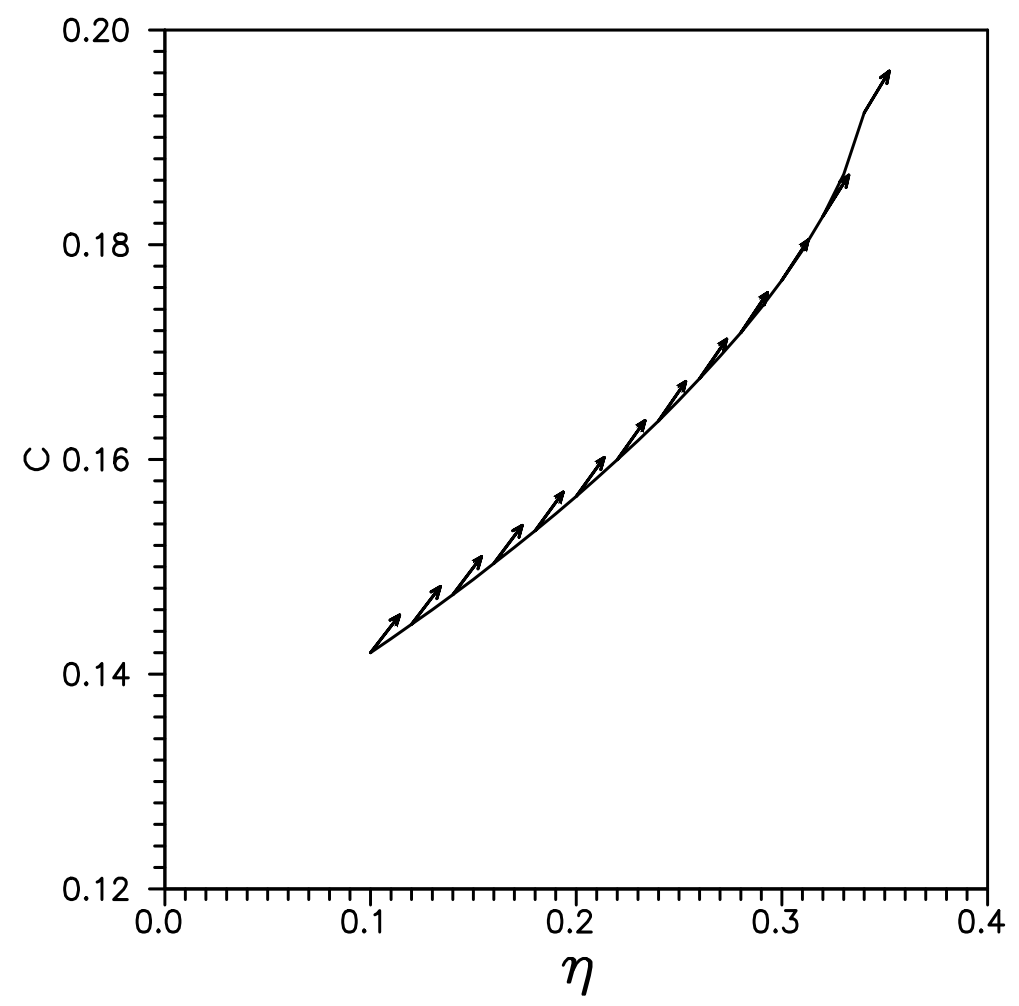

Figure 5. Same as figure 3 at $\alpha=0.9, q=0.9$ and $r=0.8$

\section{The microscopic Ginzburg-Landau-Wilson Hamiltonian in the vicinity of the binary mixture critical point}

We pass in (2.6) from $\mathrm{CV} \rho_{\vec{k}}$ and $c_{\vec{k}}$ to $\mathrm{CV} \eta_{\vec{k}}$ and $\xi_{\vec{k}}$ :

$$
\Xi_{1}=\int(d \eta)(d \xi) \exp \left[\eta_{0} \tilde{\mu}_{1}+\xi_{0} \tilde{\mu}_{2}-\frac{1}{2} \sum_{\vec{k}}\left[\eta_{\vec{k}} \eta_{-\vec{k}} P(k)+\right.\right.
$$




$$
\left.\left.\xi_{\vec{k}} \xi_{-\vec{k}} R(k)+2 \xi_{\vec{k}} \eta_{-\vec{k}} Q(k)\right]\right] J(\eta, \xi)
$$

where

$$
\begin{gathered}
\tilde{\mu}_{1}=\beta\left(A \mu_{1}^{+}+C \mu_{1}^{-}\right), \quad \tilde{\mu}_{2}=\beta\left(B \mu_{1}^{+}+D \mu_{1}^{-}\right) \\
P(k)=\frac{\beta}{V}\left(A^{2} \tilde{V}(k)+C^{2} \tilde{W}(k)+2 A C \tilde{U}(k)\right) \\
R(k)=\frac{\beta}{V}\left(B^{2} \tilde{V}(k)+D^{2} \tilde{W}(k)+2 B D \tilde{U}(k)\right) \\
Q(k)=\frac{\beta}{V}(A B \tilde{V}(k)+C D \tilde{W}(k)+(A D+B C) \tilde{U}(k)) \\
J(\eta, \xi)=\int(d \chi)(d \vartheta) \exp \left[i 2 \pi \sum_{\vec{k}}\left(\eta_{\vec{k}} \chi_{\vec{k}}+\xi_{\vec{k}} \vartheta_{\vec{k}}\right)+\sum_{n \geq 1} \bar{D}_{n}(\chi, \vartheta)\right. \\
\bar{D}_{n}(\chi, \vartheta)=\frac{(-i 2 \pi)^{n}}{n !} \sum_{\vec{k}_{1} \ldots \vec{k}_{n}} \bar{M}_{n}^{\left(i_{n}\right)}(0, \ldots) \times \\
\vartheta_{\vec{k}_{1}} \vartheta_{\vec{k}_{2}} \ldots \vartheta_{\vec{k}_{n-i_{n}}} \chi_{\vec{k}_{n-\left(i_{n+1}\right)} \ldots \chi_{\vec{k}_{n}}} \\
\chi_{\vec{k}}=A \omega_{\vec{k}}+C \gamma_{\vec{k}}, \quad \vartheta_{\vec{k}}=B \omega_{\vec{k}}+D \gamma_{\vec{k}}
\end{gathered}
$$

and $\bar{M}_{n}^{\left(i_{n}\right)}$ are linear combinations of cumulants $M_{n}^{\left(i_{n}\right)}$.

The square form in (4.1) is diagonal if

$$
Q \equiv 0
$$

Taking into account formulas from Appendix B, equation (4.8) can be rewritten in the form:

$$
\frac{(1-x) S_{a a}-x S_{b b}}{\sqrt{x(1-x)} S_{a b}}-\frac{q-1}{r}=0,
$$

where $S_{i j}(k)$ is a two-particle partial structure factor of the RS.

On the other hand, the square form in (4.7) is diagonal if

$$
\bar{M}_{2}^{(1)} \equiv 0
$$

and the last equality holds if (4.8) holds. Equation (4.8) holds in the following cases:

- a symmetrical mixture: $S_{a a}=S_{b b}, x=1 / 2, q=1$

- a non-symmetrical mixture: $(1-x) S_{a a}-x S_{b b}=0, q=1$

- a non-symmetrical mixture: $q \neq 1$. 
Let us eliminate the linear term in (4.7) by the shift

$$
\xi_{\vec{k}}=\xi_{\vec{k}}^{\prime}+\bar{M}_{1}^{(0)} \delta_{\vec{k}}
$$

and present $\bar{D}_{n}(\chi, \vartheta)$ as a sum of two terms:

$$
\bar{D}_{n}(\chi, \vartheta)=\bar{D}_{n}^{\prime}+\bar{D}_{n}^{\prime \prime}
$$

where

$$
\bar{D}_{n}^{\prime}=\bar{D}_{n}(\vartheta)+\bar{D}_{n}(\chi, \vartheta), \quad \bar{D}_{n}^{\prime \prime}=\bar{D}_{n}(\chi) .
$$

Here $D_{n}(\vartheta)$ includes only the products of variables $\vartheta_{\vec{k}}, \bar{D}_{n}(\chi, \vartheta)$ includes the mixed products of both variables $\vartheta_{\vec{k}}$ and $\chi_{\vec{k}}, \bar{D}_{n}(\chi)$ includes only the products of variables $\chi_{\vec{k}}$. Let us consider the integral

$$
\begin{aligned}
\mathcal{I}= & \int(d \xi) \exp \left[\mathcal{M}^{+} \xi_{0}-\frac{1}{2} \sum_{\vec{k}} \xi_{\vec{k}} \xi_{-\vec{k}} R(k)+i 2 \pi \sum_{\vec{k}} \xi_{\vec{k}} \vartheta_{\vec{k}}+\right. \\
& \left.\frac{(-i 2 \pi)^{2}}{2 !} \sum_{\vec{k}} \bar{M}_{2}^{(0)} \vartheta_{\vec{k}} \vartheta_{-\vec{k}}\right]\left[1+\mathcal{A}+\frac{1}{2} \mathcal{A}^{2}+\ldots\right]
\end{aligned}
$$

where the following notations are introduced:

$$
\mathcal{M}^{+}=\tilde{\mu}_{2}-R(0) \bar{M}_{1}^{(0)}, \quad \mathcal{A}=\sum_{n \geq 3} \bar{D}_{n}^{\prime},
$$

(in (4.11) the prime on $\xi_{\vec{k}}$ is omitted for clarity).

If operator $\frac{\partial}{\partial \xi_{\vec{k}}}$ is substituted for $i 2 \pi \vartheta_{\vec{k}}$, (4.12) can be rewritten as

$$
\mathcal{I}=\Xi_{\xi}^{G}\left(1+\langle\hat{\mathcal{A}}\rangle+\frac{1}{2}\left\langle\hat{\mathcal{A}}^{2}\right\rangle+\ldots\right)
$$

where

$$
\begin{gathered}
\langle\ldots\rangle=\frac{1}{\Xi_{\xi}^{G}} \frac{1}{2} \prod_{\vec{k}}\left(\pi \bar{M}_{2}^{(0)}(k)\right)^{-\frac{1}{2}} \exp (\mathcal{F}) \int(d \xi) \exp \left[\mathcal{M}^{+} \xi_{0}-\right. \\
\left.\frac{1}{2} \sum_{\vec{k}} \xi_{\vec{k}} \xi_{-\vec{k}} R(k)\right] \ldots \exp \left[-\frac{1}{2} \sum_{\vec{k}} \frac{\xi_{\vec{k}} \xi_{-\vec{k}}}{\left.\bar{M}_{2}^{(0)}\right]}\right. \\
\Xi_{\xi}^{G}=\exp (\mathcal{F}) \prod_{\vec{k}} \sqrt{R(k) \bar{M}_{2}^{(0)}(k)+1} \exp \left(\frac{\left(\bar{M}^{+}\right)^{2} \bar{M}_{2}^{(0)}}{2\left(R(0) \bar{M}_{2}^{(0)}+1\right)}\right), \\
\mathcal{F}=\bar{M}_{1}^{(0)}\left(\mathcal{M}^{+}+\frac{1}{2} R(0) \bar{M}_{1}^{(0)}\right)
\end{gathered}
$$

Finally, after the integration in (4.1) over variables $\xi_{\vec{k}}$ we obtain

$$
\Xi=\Xi_{0} \Xi_{\xi}^{G} \Delta^{\xi} \int(d \eta) \exp \left[\tilde{\mu}_{1} \eta_{0}-\frac{1}{2} \sum_{\vec{k}} \eta_{\vec{k}} \eta_{-\vec{k}} P(k)\right] J(\eta),
$$


where $\Delta^{\xi}$ is the result of integrating over $\xi_{\vec{k}}$ which does not include $\chi_{\vec{k}} . J(\eta)$ has the form:

$$
\begin{aligned}
J(\eta)= & \int(d \chi) \exp \left[i 2 \pi \sum_{\vec{k}} \chi_{\vec{k}} \eta_{\vec{k}}-i 2 \pi M_{1}(0) \sum_{\vec{k}} \chi_{\vec{k}} \delta_{\vec{k}}+\frac{(-i 2 \pi)^{2}}{2 !} M_{2}(0) \sum_{\vec{k}} \chi_{\vec{k}} \chi_{-\vec{k}}+\right. \\
& \frac{(-i 2 \pi)^{3}}{3 !} M_{3}(0, \ldots) \sum_{\vec{k}_{1} \vec{k}_{2} \vec{k}_{3}} \chi_{\vec{k}_{1}} \chi_{\vec{k}_{2}} \chi_{\vec{k}_{3}} \delta_{\vec{k}_{1}+\vec{k}_{2}+\vec{k}_{3}}+\frac{(-i 2 \pi)^{4}}{4 !} M_{4}(0, \ldots) \times \\
& \sum_{\vec{k}_{1} \vec{k}_{2} \vec{k}_{3} \vec{k}_{4}} \chi_{\vec{k}_{1}} \chi_{\vec{k}_{2}} \chi_{\vec{k}_{3}} \chi_{\vec{k}_{4}} \delta_{\left.\vec{k}_{1}+\vec{k}_{2}+\vec{k}_{3}+\vec{k}_{4}\right] .}
\end{aligned}
$$

Here

$$
M_{n}(0, \ldots)=\bar{M}_{n}^{(n)}(0, \ldots)+\triangle M_{n}(0, \ldots) .
$$

Setting $\mathcal{M}^{+}=0$ we get the following expressions for $\triangle M_{n}(0, \ldots)$ :

$$
\begin{aligned}
& \Delta M_{1}=\frac{\bar{M}_{3}^{(1)}}{2\langle N\rangle} \sum_{\vec{k}^{\prime}} \tilde{g}\left(k^{\prime}\right)+\ldots \\
& \Delta M_{2}= \frac{\bar{M}_{4}^{(2)}}{2 !\langle N\rangle} \sum_{\vec{k}^{\prime}} \tilde{g}\left(k^{\prime}\right)+\frac{\left(\bar{M}_{3}^{(1)}\right)^{2}}{2\langle N\rangle^{2}} \sum_{\vec{k}^{\prime}} \tilde{g}\left(k^{\prime}\right) \tilde{g}\left(\left|\vec{k}-\vec{k}^{\prime}\right|\right)+ \\
& \frac{\left(\bar{M}_{4}^{(1)}\right)^{2}}{3 !\langle N\rangle^{3}} \sum_{\vec{k}^{\prime} \vec{k}^{\prime \prime}} \tilde{g}\left(k^{\prime}\right) \tilde{g}\left(k^{\prime \prime}\right) \tilde{g}\left(\left|\vec{k}-\vec{k}^{\prime}-\vec{k}^{\prime \prime}\right|\right)+\ldots \\
& \triangle M_{3}= \frac{3}{2} \bar{M}_{3}^{(2)} \bar{M}_{4}^{(1)} \frac{1}{\langle N\rangle} \tilde{g}\left(k_{2}\right) \frac{1}{\langle N\rangle} \sum_{\vec{k}^{\prime}} \tilde{g}\left(k^{\prime}\right)+\frac{3}{2} \bar{M}_{3}^{(1)} \bar{M}_{4}^{(2)} \times \\
& \frac{1}{\langle N\rangle^{2}} \sum_{\vec{k}^{\prime}} \tilde{g}\left(k^{\prime}\right) \tilde{g}\left(\left|\vec{k}_{2}+\vec{k}_{3}-\vec{k}^{\prime}\right|\right)+\ldots \\
& \triangle M_{4}= 3\left(\bar{M}_{3}^{(2)}\right)^{2} \frac{1}{\langle N\rangle} \tilde{g}\left(\left|\vec{k}_{3}+\vec{k}_{4}\right|\right)+2 \bar{M}_{4}^{(1)} \bar{M}_{4}^{(3)} \frac{1}{\langle N\rangle^{2}} \times \\
& \sum_{\vec{k}^{\prime}} \tilde{g}\left(k^{\prime}\right) \tilde{g}\left(\left|\overrightarrow{k_{2}}+\vec{k}_{3}+\vec{k}_{4}\right|\right)+\frac{3}{2}\left(\bar{M}_{4}^{(2)}\right)^{2} \frac{1}{\langle N\rangle^{2}} \times \\
& \sum_{\vec{k}^{\prime}} \tilde{g}\left(k^{\prime}\right) \tilde{g}\left(\left|\vec{k}_{3}+\vec{k}_{4}-\vec{k}^{\prime}\right|\right)+\ldots,
\end{aligned}
$$

where

$$
\begin{gathered}
\tilde{g}(k)=-\frac{\tilde{R}(k)}{1+\tilde{R}(k) \bar{S}_{2}^{(0)}} \\
\tilde{R}(k)=R(k)\langle N\rangle, \quad \bar{S}_{2}^{(0)}=\bar{M}_{2}^{(0)} /\langle N\rangle .
\end{gathered}
$$


An estimation of corrections $\triangle M_{n}$ was carried out for the symmetrical mixture in [ 37. In this case

$$
M_{2}=1+\delta_{1}, \quad M_{3}=0, \quad M_{4}=-2+\delta_{2},
$$

where $\delta_{i}$ are small values.

Let us consider formula (4.3) for $P(k)$. Substituting into (4.3) coefficients $A, B, C$ and $D$ from Appendix B, we get

$$
\begin{aligned}
P(k)= & \frac{\beta}{V}\left[\frac{1}{2}\left(\tilde{\phi}_{a a}(k)+\tilde{\phi}_{b b}(k)\right)+\frac{1}{\sqrt{4 A_{12}^{2}+\left(A_{11}-A_{22}\right)^{2}}} \times\right. \\
& {\left.\left[\tilde{\phi}_{a b}(k)\left(A_{11}-A_{22}\right)+\left(\tilde{\phi}_{a a}(k)-\tilde{\phi}_{b b}(k)\right) A_{12}\right]\right], }
\end{aligned}
$$

where $A_{11}, A_{22}$ and $A_{12}$ are functions of temperature. Using condition (4.8), $P(k)$ can be represented as

$$
\begin{aligned}
\left.P(k)\right|_{Q=0}= & \frac{\beta}{2 V}\left[\tilde{\phi}_{a a}(k)+\tilde{\phi}_{b b}(k)+2 \frac{A_{11}-A_{22}}{\left|A_{11}-A_{22}\right|} \frac{1}{\sqrt{1+\kappa^{2}}} \times\right. \\
& \left(\tilde{\phi}_{a b}(k)+\kappa\left(\tilde{\phi}_{a a}(k)-\tilde{\phi}_{b b}(k)\right)\right],
\end{aligned}
$$

where

$$
\kappa=\frac{q-1}{r}=\frac{(1-x) S_{a a}-x S_{b b}}{2 \sqrt{x(1-x)} S_{a b}} .
$$

$P(k)$ takes both negative ( at small $|\vec{k}|$ ) and positive ( at large $|\vec{k}|$ ) values. In the region $|\vec{k}|>B$ (see Fig. 6) we can integrate in (4.15) over $\chi_{\vec{k}}$ and $\eta_{\vec{k}}$ with the Gaussian measure density as the basic one. As a result, we get in the approximation of the $\eta^{4}$ model

$$
\begin{aligned}
\Xi= & \Xi_{0} \Xi_{\xi}^{G} \Delta^{\xi} \Delta^{\eta} \int d(\eta)^{N_{B}} d(\chi)^{N_{B}} \exp \left[\tilde{\mu}_{1} \eta_{0}-\frac{1}{2} \sum_{\vec{k}} P(k) \eta_{\vec{k}} \eta_{-\vec{k}}+\right. \\
& i 2 \pi \sum_{\vec{k}} \chi_{\vec{k}} \eta_{\vec{k}}-i 2 \pi \bar{M}_{1}(0) \sum_{\vec{k}} \chi_{\vec{k}} \delta_{\vec{k}}+\frac{(-i 2 \pi)^{2}}{2 !} \bar{M}_{2}(0) \sum_{\vec{k}} \chi_{\vec{k}} \chi_{-\vec{k}}+ \\
& \frac{(-i 2 \pi)^{3}}{3 !} \bar{M}_{3}(0, \ldots) \sum_{\vec{k}_{1} \vec{k}_{2} \vec{k}_{3}} \chi_{\vec{k}_{1}} \chi_{\vec{k}_{2}} \chi_{\vec{k}_{3}} \delta_{\vec{k}_{1}+\vec{k}_{2}+\vec{k}_{3}}+\frac{(-i 2 \pi)^{4}}{4 !} \bar{M}_{4}(0, \ldots) \times \\
& \sum_{\vec{k}_{1} \vec{k}_{2} \vec{k}_{3} \vec{k}_{4}} \chi_{\vec{k}_{1}} \chi_{\vec{k}_{2}} \chi_{\vec{k}_{3}} \chi_{\vec{k}_{4}} \delta_{\left.\vec{k}_{1}+\vec{k}_{2}+\vec{k}_{3}+\vec{k}_{4}\right], \quad\left|\vec{k}_{i}\right|<B}
\end{aligned}
$$

where in place of $M_{i}(0, \ldots)$ stand renormalized coefficients $\bar{M}_{i}(0, \ldots)$ :

$$
\begin{aligned}
\bar{M}_{i}(0, \ldots) & =M_{i}(0, \ldots)+\Delta \bar{M}_{i}(0, \ldots) \\
\Delta^{\eta} & =\prod_{|\vec{k}|>B} \sqrt{P(k) \bar{M}_{2}+1}
\end{aligned}
$$


We can consider a set of $\vec{k}$ vectors, $|\vec{k}|<B$, as corresponding to the sites of a reciprocal lattice conjugated to a certain block lattice $r_{l}$ with $N_{B}$ block sites in volume $V$ :

$$
\left\langle N_{B}\right\rangle=V(B / \pi)^{3}=\frac{\langle N\rangle\left(B \sigma_{b b}\right)^{3}\left(x+\alpha^{3}(1-x)\right)}{6 \pi^{2} \eta} .
$$

One may consider quantity $B$ as the size of the first Brillouin zone of this block lattice.

Next, two shifts are carried out in order to eliminate the cubic term in (4.23) 34:

$$
\chi_{\vec{k}}=\chi_{\vec{k}}^{\prime}+\Delta \delta_{\vec{k}}, \quad \eta_{\vec{k}}=\eta_{\vec{k}}^{\prime}+\tilde{M}_{1} \delta_{\vec{k}}
$$

where

$$
\Delta=-i 2 \pi \bar{M}_{3}
$$

and

$$
\tilde{M}_{1}=\bar{M}_{1}-\frac{\bar{M}_{2} \bar{M}_{3}}{\bar{M}_{4}}+\frac{1}{3} \frac{\left(\bar{M}_{3}\right)^{3}}{\left(\bar{M}_{4}\right)^{2}} .
$$

Then, (4.23) has the form:

$$
\begin{aligned}
\Xi= & \Xi_{0} \Xi_{\xi}^{G} \Delta^{\xi} \Delta^{\eta} \exp (\mathcal{G}) \int d\left(\eta^{\prime}\right)^{N_{B}} d\left(\chi^{\prime}\right)^{N_{B}} \exp \left[h \eta_{0}^{\prime}-\frac{1}{2} \sum_{\vec{k}} P(k) \times\right. \\
& \eta_{\vec{k}}^{\prime} \eta_{-\vec{k}}^{\prime}+i 2 \pi \sum_{\vec{k}} \chi_{\vec{k}}^{\prime} \eta_{\vec{k}}^{\prime}+\frac{(-i 2 \pi)^{2}}{2 !} \tilde{M}_{2}(0) \sum_{\vec{k}} \chi_{\vec{k}}^{\prime} \chi_{-\vec{k}}^{\prime}+\frac{(-i 2 \pi)^{4}}{4 !} \times \\
& \left.\tilde{M}_{4}(0, \ldots) \sum_{\vec{k}_{1} \vec{k}_{2} \vec{k}_{3} \vec{k}_{4}} \chi_{\vec{k}_{1}}^{\prime} \chi_{\vec{k}_{2}}^{\prime} \chi_{\vec{k}_{3}}^{\prime} \chi_{\vec{k}_{4}}^{\prime} \delta_{\vec{k}_{1}+\vec{k}_{2}+\vec{k}_{3}+\vec{k}_{4}}\right], \quad\left|\vec{k}_{i}\right|<B,
\end{aligned}
$$

where

$$
\begin{gathered}
\tilde{M}_{2}(0)=\bar{M}_{2}(0)-\frac{1}{2} \frac{\left(\bar{M}_{3}(0)\right)^{2}}{\bar{M}_{4}(0)}, \quad \tilde{M}_{4}(0, \ldots)=\bar{M}_{4}(0, \ldots) \\
\mathcal{G}=-\frac{\bar{M}_{3}}{\bar{M}_{4}}\left(\bar{M}_{1}-\frac{\bar{M}_{2} \bar{M}_{3}}{2 \bar{M}_{4}}+\frac{1}{8} \frac{\left(\bar{M}_{3}\right)^{3}}{\left(\bar{M}_{3}\right)^{2}}\right)+\tilde{M}_{1}\left(\tilde{\mu}_{1}-\frac{1}{2} \tilde{M}_{1}\right)+\frac{\tilde{M}_{1} \bar{M}_{3}}{\bar{M}_{4}} \\
h=\tilde{\mu}_{1}-P(0) \tilde{M}_{1}+\frac{\bar{M}_{3}}{\bar{M}_{4}}
\end{gathered}
$$

After the integration over $\chi_{\vec{k}}^{\prime}$ in (4.24) we get

$$
\Xi=C \int \exp \left[E_{4}(\eta)\right](d \eta)^{N_{B}}
$$

where

$$
\begin{aligned}
& C=\Xi_{0} \Xi_{\xi}^{G} \Delta^{\xi} \Delta^{\eta} \exp \left(\mathcal{G}+a_{0} N_{B}\right) \sqrt{2}^{N_{B}-1} \\
& E_{4}(\eta)= h \eta_{0}-\frac{1}{2\left\langle N_{B}\right\rangle} \sum_{\vec{k}} d_{2}(k) \eta_{\vec{k}} \eta_{-\vec{k}}- \\
& \frac{a_{4}}{4 !\left\langle N_{B}\right\rangle^{3}} \sum_{\vec{k}_{1} \ldots \vec{k}_{4}} \eta_{\vec{k}_{1}} \eta_{\vec{k}_{2}} \eta_{\vec{k}_{3}} \eta_{\vec{k}_{4}} \delta_{\vec{k}_{1}+\vec{k}_{2}+\vec{k}_{3}+\vec{k}_{4}, \quad\left|\vec{k}_{i}\right|<B}
\end{aligned}
$$




$$
\begin{gathered}
a_{0}=\ln \left[\frac{1}{\sqrt{\pi}}\left(\frac{N_{B}}{N}\right)^{1 / 4}\left(\frac{3}{\left|\tilde{S}_{4}\right|}\right)^{1 / 4} \exp \left(\frac{z^{2}}{4}\right) U(0, z)\right] \\
d_{2}(k)=a_{2}+P(k) \\
a_{2}=\sqrt{\frac{12}{\left|\tilde{S}_{4}\right|} \frac{\left\langle N_{B}\right\rangle}{\langle N\rangle} \mathcal{K}(z)} \\
a_{4}=36 \frac{\left\langle N_{B}\right\rangle}{\langle N\rangle} \frac{1}{\left|\tilde{S}_{4}\right|}\left[\mathcal{K}^{2}(z)+\frac{2}{3} \mathcal{K}(z)-\frac{2}{3}\right] \\
\mathcal{K}(z)=U(1, z) / U(0, z) \\
z=\tilde{S}_{2} \sqrt{\frac{3}{\left|\tilde{S}_{4}\right|} \frac{\langle N\rangle}{\left\langle N_{B}\right\rangle}} \\
\tilde{S}_{n}=\sqrt{2}{ }^{n} \tilde{M}_{n} /\langle N\rangle .
\end{gathered}
$$

Here $U(a, z)$ is the parabolic cylinder function.

As it is seen from (4.27), $E_{4}(\eta)$ has the form analogous to the basic density measure of the $3 D$ Ising model in an external field [32]. But the main difference is the dependence of coefficients $a_{0}, a_{2}$ and $a_{4}$ (see (4.28)-(4.33)) on the microscopic parameters of the system.

\section{Conclusions}

We propose the microscopic approach to the study of phase transitions and critical phenomena in multicomponent mixtures. It is based on the CV method with RS. This method allows us to take into account the short-range and long-range interactions simultaneously.

We consider the task of the definition of the order parameter in a binary mixture and show that it has a consistent and clear solution within the framework of our approach.

After integration over $\mathrm{CV} \xi_{\vec{k}}$ (which do not include the variable connected with the order parameter) the basic density measure with respect to CV $\eta_{\vec{k}}$ (GinsburgLandau-Wilson Hamiltonian) is constructed. It is shown that the task can be reduced to the 3D Ising model.

\section{Appendix A}

A grand partition function of a two-component fluid system in the CV representation with the RS can be written as [36]:

$$
\Xi=\Xi_{0} \Xi_{1}
$$

where

$$
\Xi_{0}=\sum_{N_{a}=0}^{\infty} \sum_{N_{b}=0}^{\infty} \prod_{\gamma=a}^{b} \exp \left[\frac{\beta \mu_{0}^{\gamma} N_{\gamma}}{N_{\gamma} !}\right] \int(d \Gamma) \exp \left[-\frac{\beta}{2} \sum_{\gamma \delta} \sum_{i j} \psi_{\gamma \delta}\left(r_{i j}\right)\right]
$$


is the grand partition function of the $\mathrm{RS} ; \beta=\frac{1}{k T}$ is the reciprocal temperature; $(d \Gamma)=\prod_{a, b} d \Gamma_{N_{\gamma}}, d \Gamma_{N_{\gamma}}=d \vec{r}_{1}^{\gamma} d \vec{r}_{2}^{\gamma} \ldots d \vec{r}_{N_{\gamma}}^{\gamma}$ is an element of the configurational space of the $\gamma$-th species; $\mu_{0}^{\gamma}$ is the chemical potential of the $\gamma$-th species in the RS.

The part of the grand partition function which is defined in the CV phase space has the form of the functional integral:

$$
\Xi_{1}=\int(d \rho) \exp \left[\beta \sum_{\gamma} \mu_{1}^{\gamma} \rho_{0, \gamma}-\frac{\beta}{2 V} \sum_{\gamma \delta} \sum_{\vec{k}} \tilde{\phi}_{\gamma \delta}(k) \rho_{\vec{k}, \gamma} \rho_{-\vec{k}, \delta}\right] J\left(\rho_{a}, \rho_{b}\right) .
$$

Here,

1) $\mu_{1}^{\gamma}$ is a part of the chemical potential of the $\gamma$-th species

$$
\mu_{1}^{\gamma}=\mu_{\gamma}-\mu_{0}^{\gamma}+\frac{\beta}{2 v} \sum_{\vec{k}} \tilde{\phi}_{\gamma \gamma}(k)
$$

and is determined from the equation

$$
\frac{\partial \ln \Xi_{1}}{\partial \beta \mu_{1}^{\gamma}}=\left\langle N_{\gamma}\right\rangle
$$

$\mu_{\gamma}$ is the full chemical potential of the $\gamma$-th species;

2) $\rho_{\vec{k}, \gamma}=\rho_{\vec{k}, \gamma}^{c}-i \rho_{\vec{k}, \gamma}^{s}$ is the collective variable of the $\gamma$-th species, the indices $c$ and $s$ denote the real part and the coefficient at the imaginary part of $\rho_{\vec{k}, \gamma} ; \rho_{\vec{k}, \gamma}^{c}$ and $\rho_{\vec{k}, \gamma}^{s}$ describe the value of $\vec{k}$-th fluctuation mode of the number of $\gamma$-th species particles. Each of $\rho_{\vec{k}, \gamma}^{c}$ and $\rho_{\vec{k}, \gamma}^{s}$ takes all the real values from $-\infty$ to $+\infty$. $(d \rho)$ is a volume element of the $\mathrm{CV}$ phase space:

$$
(d \rho)=\prod_{\gamma} d \rho_{0, \gamma} \prod_{\vec{k} \neq 0}^{\prime} d \rho_{\vec{k}, \gamma}^{c} d \rho_{\vec{k}, \gamma}^{s} .
$$

The prime means that the product over $\vec{k}$ is performed in the upper semispace;

3) $J\left(\rho_{a}, \rho_{b}\right)$ is the transition Jacobian to the CV averaged on the RS:

$$
\begin{aligned}
J\left(\rho_{a}, \rho_{b}\right)= & \int(d \nu) \prod_{\gamma=a}^{b} \exp \left[i 2 \pi \sum_{\vec{k}} \nu_{\vec{k}, \gamma} \rho_{\vec{k}, \gamma}\right] \exp \left[\sum_{n \geq 1} \frac{(-i 2 \pi)^{n}}{n !} \times\right. \\
& \left.\sum_{\gamma_{1} \ldots \gamma_{n}} \sum_{\vec{k}_{1} \ldots \vec{k}_{n}} M_{\gamma_{1} \ldots \gamma_{n}}\left(\vec{k}_{1}, \ldots, \vec{k}_{n}\right) \nu_{\vec{k}_{1}, \gamma_{1}} \ldots \nu_{\vec{k}_{n}, \gamma_{n}}\right]
\end{aligned}
$$

where variable $\nu_{\vec{k}, \gamma}$ is conjugated to $\mathrm{CV} \rho_{\vec{k}, \gamma} . M_{\gamma_{1} \ldots \gamma_{n}}\left(\vec{k}_{1}, \ldots, \vec{k}_{n}\right)$ is the $n$-th cumulant connected with $S_{\gamma_{1} \ldots \gamma_{n}}\left(k_{1}, \ldots, k_{n}\right)$, the $n$-particle partial structure factor of the RS, by means of the relation

$$
M_{\gamma_{1} \ldots \gamma_{n}}\left(\vec{k}_{1}, \ldots, \vec{k}_{n}\right)=\sqrt[n]{N_{\gamma_{1}} \ldots N_{\gamma_{n}}} S_{\gamma_{1} \ldots \gamma_{n}}\left(k_{1}, \ldots, k_{n}\right) \delta_{\vec{k}_{1}+\cdots+\vec{k}_{n}}
$$

where $\delta_{\vec{k}_{1}+\cdots+\vec{k}_{n}}$ is the Kroneker symbol. 
In general, the dependence of $M_{\gamma_{1} \ldots \gamma_{n}}\left(\vec{k}_{1}, \ldots, \vec{k}_{n}\right)$ on wave vectors $\vec{k}_{1}, \ldots, \vec{k}_{n}$ is complicated. Hereafter we shall replace $M_{\gamma_{1} \ldots \gamma_{n}}\left(\vec{k}_{1}, \ldots, \vec{k}_{n}\right)$ by their values in longwave length limit $M_{\gamma_{1} \ldots \gamma_{n}}(0, \ldots, 0)$;

4) $\tilde{\phi}_{\gamma \delta}(k)$ is the Fourier transform of attractive potential $\phi_{\gamma \delta}(r)$. Function $\tilde{\phi}_{\gamma \delta}(k)$ satisfies the following requirements: $\tilde{\phi}_{\gamma \delta}(k)$ is negative for small values of $\vec{k}$ and $\lim _{\vec{k} \rightarrow \infty} \tilde{\phi}_{\gamma \delta}(k)=0$. The behaviour of $\phi_{\gamma \delta}(r)$ in the region of the core $r<\sigma_{\gamma \delta}$ must be determined from the conditions of optimal separation of the interaction. For a very broad class of potentials the general form of $\tilde{\phi}_{\gamma \delta}(k)$ is presented in figure 6 .

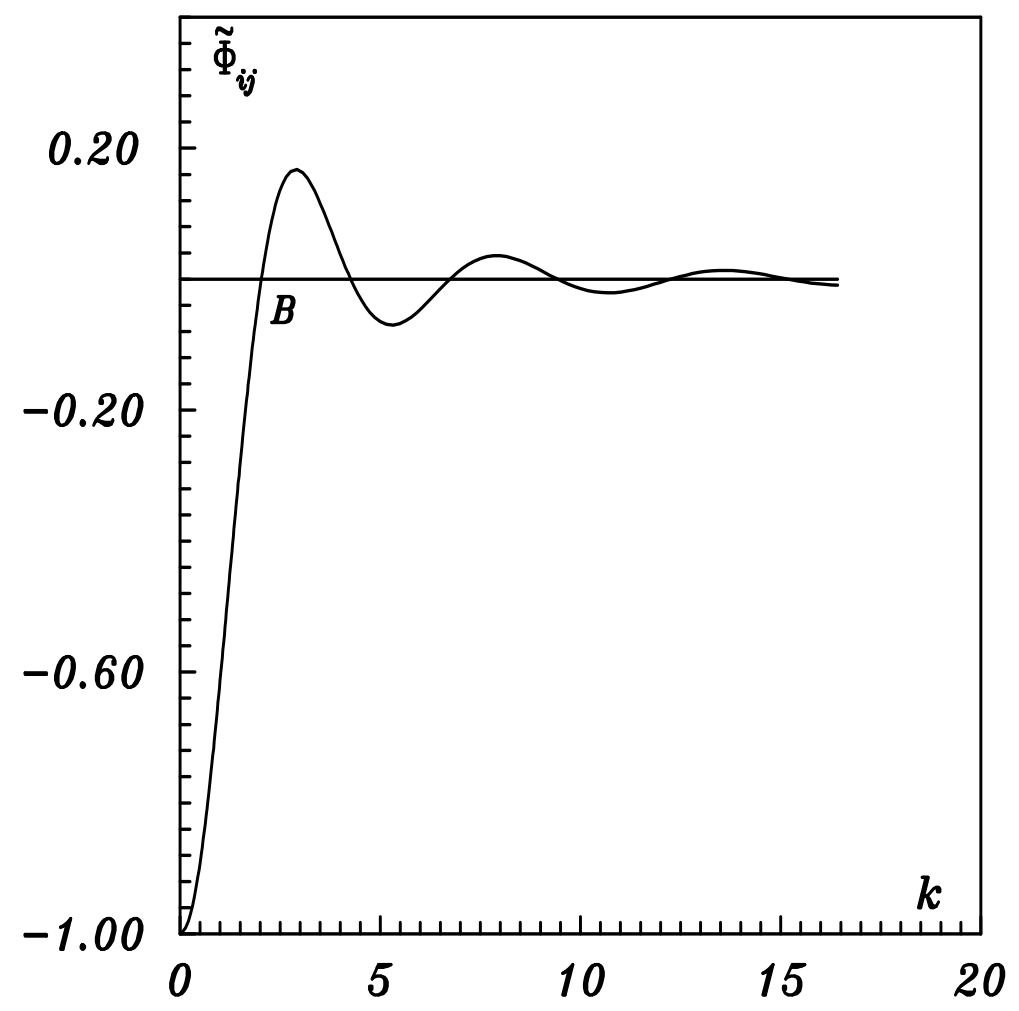

Figure 6. The Fourier transform of the attractive potential $\phi_{\gamma \delta}(r)$

We pass in (5.1) to CV $\rho_{\vec{k}}$ and $c_{\vec{k}}$ (according to $\omega_{\vec{k}}$ and $\gamma_{\vec{k}}$ ) by means of the orthogonal linear transformation:

$$
\begin{gathered}
\rho_{\vec{k}}=\frac{\sqrt{2}}{2}\left(\rho_{\vec{k}, a}+\rho_{\vec{k}, b}\right) \\
c_{\vec{k}}=\frac{\sqrt{2}}{2}\left(\rho_{\vec{k}, a}-\rho_{\vec{k}, b}\right) .
\end{gathered}
$$

As a result, for $\Xi_{1}$ we obtain formulas (2.1)-(2.7). 


\section{Appendix B}

The coefficients $A(k), B(k), C(k)$ and $D(k)$ have the forms:

$$
\begin{aligned}
A= & \sqrt{2}\left|A_{12}\right|\left[4 A_{12}^{2}+\left(A_{11}-A_{22}\right)^{2}-\left(A_{11}-A_{22}\right) \times\right. \\
& \left.\sqrt{\left(A_{11}-A_{22}\right)^{2}+4 A_{12}^{2}}\right]^{-1}, \\
B= & \sqrt{2}\left|A_{12}\right|\left[4 A_{12}^{2}+\left(A_{11}-A_{22}\right)^{2}+\left(A_{11}-A_{22}\right) \times\right. \\
& \sqrt{\left.\left(A_{11}-A_{22}\right)^{2}+4 A_{12}^{2}\right]^{-1}}, \\
C= & -\frac{\sqrt{2}}{2} \frac{\left|A_{12}\right|}{\left(A_{12}\right)}\left[A_{11}-A_{22}-\sqrt{\left(A_{11}-A_{22}\right)^{2}+4 A_{12}^{2}}\right] \times \\
& {\left[4 A_{12}^{2}+\left(A_{11}-A_{22}\right)^{2}-\left(A_{11}-A_{22}\right) \sqrt{\left(A_{11}-A_{22}\right)^{2}+4 A_{12}^{2}}\right]^{-1}, } \\
D= & -\frac{\sqrt{2}}{2} \frac{\left|A_{12}\right|}{\left(A_{12}\right)}\left[A_{11}-A_{22}+\sqrt{\left(A_{11}-A_{22}\right)^{2}+4 A_{12}^{2}}\right] \times \\
& {\left[4 A_{12}^{2}+\left(A_{11}-A_{22}\right)^{2}+\left(A_{11}-A_{22}\right) \sqrt{\left(A_{11}-A_{22}\right)^{2}+4 A_{12}^{2}}\right]^{-1} . }
\end{aligned}
$$

\section{Appendix C}

The Helmholtz free energy of a binary mixture in the mean field approximation can be written as

$$
f_{m f}=f_{i d}+f_{r e f}+f_{\text {attr }}
$$

where $f_{i d}$ is the free energy of a binary mixture of ideal gases, $f_{r e f}$ is the free energy of a binary mixture of hard spheres [ 45]:

$$
\begin{gathered}
f_{\text {ref }}=F_{\text {ref }} /\langle N\rangle k_{B} T=-1.5\left(1-y_{1}+y_{2}+y_{3}\right)+\left(3 y_{2}+2 y_{3}\right)(1-\eta)^{-1} \\
+1.5\left(1-y_{1}-y_{2}-y_{3} / 3\right)(1-\eta)^{-2}+\left(y_{3}-1\right) \ln (1-\eta), \\
y_{1}=\Delta_{12} \frac{1+\alpha}{\sqrt{\alpha}}, \quad y_{2}=\Delta_{12} \frac{\eta_{a} \alpha+\eta_{b}}{\sqrt{\alpha} \eta} \\
\Delta_{12}=\frac{\sqrt{\eta_{a} \eta_{b}}}{\eta} \frac{(\alpha-1)^{2}}{\alpha} \sqrt{x(1-x)} \\
\eta_{a}=\frac{(1-x) \alpha^{3} \eta}{x+(1-x) \alpha^{3}}, \quad \eta_{b}=\frac{x \eta}{x+(1-x) \alpha^{3}} .
\end{gathered}
$$

$f_{\text {attr }}=F_{\text {attr }} /\langle N\rangle k_{B} T$ is the contribution due to attraction between the particles:

$$
f_{\text {attr }}=-\frac{1}{2} \frac{\eta}{T^{*}\left(x+(1-x) \alpha^{3}\right)}\left((1-x)^{2}+2 x(1-x) r+x^{2} q\right),
$$

where $T^{*}=k_{B} T \sigma^{3}\left|\tilde{\phi}_{a a}(0)\right|^{-1} \pi / 6$ is the dimensionless temperature. 


\section{References}

1. Rowlinson J.S.: Liquids and liquid Mixtures.Butterworths-Scientific, London,1959.

2. Schneider G.M. //Adv.Chem.Phys. 1970, vol. 17, No 1, p. 1-42.

3. Kumar A., Krishamurthy H.R. and Gopal E.S.R.// Phys. Rep. 1983, vol. 98, p. 57-143.

4. Durenberg A., Schouten J.A., Trappeniers N.J.// Physica A, 1980, vol. 101, No 2-3, p. 895-899.

5. Schneider G.M.// J. Chem. Thermod. 1991, vol. 23, p. 301-326.

6. Fisher M.E., //Phys. Rev. 1968, vol. 176, p. 257-272.

7. Griffiths R.B., Wheeler J.C.// Phys. Rev.1970, vol. A2, p. 1047-1064.

8. Saam W.F.// Phys. Rev. 1970, vol. A2, p. 1461-1466.

9. Sengers J.V., Levelt Sengers J.M.H.// Ann. Rev. Phys. Chem. 1986, vol. 37, p.189-222.

10. Anisimov M.A., Gorodetskii E.E., Kulikov V.D., Sengers J.V.// Phys.Rev. E,1995, vol. 51, p. 1199-1215.

11. Anisimov M.A., Gorodetskii E.E., Kulikov V.D., Povodyrev A.A., Sengers J.V.// Physica A,1995, vol. 220, p. 277-324.

12. Van Konynenburg P.H., Scott R.L. // Phyl.Trans.R.Soc. London, Ser. A, 1980, vol. 298A, No 1442, p.495-540.

13. Waisman E. // J.Chem.Phys., 1973, vol.59, No 1, p. 495-497.

14. Jedrzejek C., Konior J., Streszewski M. // Phys. Rev. A, 1986, vol.35, No 3, p. 12261234 .

15. Arrieta E., Jedrzejek C., Marsh K.M. // J.Chem.Phys., 1987, vol.87, No 6, p.36073626 .

16. Abramo M.C., Caccamo C., Giunta G. // Phys.Rev. A, 1986, vol. 34, No 4, p. 32793287.

17. McGuigan D.B., Monson P.A. // Mol.Phys., 1987, vol. 62, No 1, p. 3-18.

18. Caccamo C., Malescio G. // Phys.Rev. A, 1989, vol.40, No 11, p. 6384-6387.

19. Malescio G. // Phys.Rev. A, 1990, vol.42, No 10, p. 6241-6242.

20. Chen X.S., Forstmann F.// J.Chem.Phys., 1992, vol.97, No 5, p. 3696-3703.

21. Malescio G. // J.Chem.Phys., 1992, vol.96, No 1, p. 648-651.

22. Parola A., Reatto L. // Phys. Rev. A., 1991, vol. 44, p. 6600-6615.

23. Parola A., Reatto L. // J. Phys.: Condens. Matter, 1993, vol. 5, p. B165-B172.

24. Parola A., Pini D., Reatto L. // J. Phys.: Condens. Matter, 1994, vol. 6,p. A167-A170.

25. Parola A.,Reatto L.// Advan. in Phys. 1995, vol. 44, No 3, p.211-298.

26. Reatto L., Parola A. // J. Phys.: Condens. Matter, 1996, vol. 8, p. 9221-9231.

27. Pini D., Reatto L.,Parola A. // J. Phys.: Condens. Matter, 1997, vol. 9, p. 1417-1423.

28. Parola A., Reatto L. //Phys.Rev. A,1985, vol. 31, No 5, p. 3309-3322.

29. Pini D., Parola A., Reatto L. // J.Stat.Phys., 1993, vol. 72, Nos. 5/6, p. 1179-1201.

30. Zubarev D.N. // DAN USSR, 1964, vol. 95, p. 757.

31. Yukhnovskii I.R., Holovko M.F.: Statistical Theory of Classical Equilibrium Systems (Naukova Dumka,Kiev,1980).p.372.

32. Yukhnovskii I.R.: Phase Transitions of the Second Order: Collective Variables Method (World Sci.Publ.Co.Ltd.Singapore,1987), p.327.

33. Yukhnovskii I.R. // Physica A, 1990, vol.168, No 3, p. 999-1020.

34. Yukhnovskii I.R.,// Proceed. of the Steclov Inst.of Math., 1992, vol. 2, p. 223.

35. Yukhnovskii I.R.,Idzyk I.M.,Kolomietc V.O,// J.Stat.Phys., 1995 , vol. 80, p.405.

36. Patsagan O.V., Yukhnovskii I.R. // Theoret.Math.Phys., 1990, vol.83, No 1, p. 387. 
37. Yukhnovskii I.R., PatsahanO.V.// J.Stat. Phys., 1995, vol. 81, p. 647.

38. Patsahan O.V. //Cond.Matt.Phys., 1995, vol. 5, p. 124.

39. Patsahan O.V.// Ukr.Fiz.Zh., 1992, vol. 37, p. 582 (in Ukrainian).

40. Patsahan O.V., The phase transitions in binary systems.I.Random phase approximation, Preprint Inst. Cond. Matter Phys. Acad. Sci. Ukraine: ICMP-92-2U, Lviv,1992.(in Ukrainian).

41. Patsahan O.V. //Ukr.Fiz.Zh., 1996, vol. 41, No 9, p. 877 (in Ukrainian).

42. Patsahan O.V., Yukhnovskii I.R. // Theor. Mat. Fiz., 1987, vol. 72, p.452.

43. Biben T.,Hansen J.-P.//Phys.Rev.Lett.-1991, vol. 66, No 17, p. 2215-2218.

44. Lebowitz J.L. // Phys.Rev., 1964, vol.133, No 4A, p.895-899.

45. Mansoori G.A., Carnahan N.F., Starling K.E., Leland T.W., Jr.//J.Chem. Phys., 1971, vol. 51, No 4, p.1523-1525. 